\title{
Molecular Tagging Velocimetry for Microfluid Applications
}

\author{
Zilan Shen, Hyoun Park, Jia Ming Chen, Yongchi Tian, and Ann-Marie Lanzillotto \\ Sarnoff Corporation, 201 Washington Road, \\ Princeton, NJ 08540
}

\begin{abstract}
We are developing a high-resolution, molecular tagging velocimetry technique that will accelerate the design and optimization of micro-scale devices such as a microtissue array based biosensor. The complex aerodynamic and hydrodynamic flows around and within the devices can be analyzed with capabilities beyond that of traditional measurement techniques. This non-invasive measurement technique will provide highly detailed information on the entire flow field, such as 3-D velocity, 3-D vorticity, 3-D. pressure fields, shear stress and flow rate distribution patterns within micro-scaled devices.
\end{abstract}

\section{INTRODUCTION}

Microfluidic technology exploits sample size reduction and parallel processing for sensor, micro-pumping and actuation applications. These applications require precise fluidic delivery and management that can only be achieved with a correct knowledge of the flow field within individual channels and valves [1]. Further more, some of the applications will also benefit from accurate measurement of the pressure and force field related to the flow media [2]. For example, viscous damping and pressure field information are crucial in evaluating the squeeze film damping effects on the dynamic responses of MEMS devices, such as resonance frequency and the Q-number of an actuator [3].

In the past, traditional techniques have not provided detailed flow information on the micro scale. Point-by-point measurement methods, including laser Doppler velocimetry (LDV) and hot wire techniques, are limited to steady or periodic flows. When used for micro-scale application, hot-film techniques are highly invasive and can create substantial flow disturbances. Similarly, the typical sample volume size for LDV has been on the order of hundreds of microns, making it very difficult to obtain near-wall measurement due to the backscatter of the laser signal from boundaries. Furthermore, these techniques are not suitable for very low velocity flow measurements. The hot-wire sensor measures the heat transfer from the environment, and therefore suffers from the "free convection" effect. Similarly, since LDV measures the Doppler frequency shift due to the movement of the scattering center (particle), more complicated heterodyne detection is needed to differentiate a very small frequency change, on the order of ten to hundred $\mathrm{Hz}$, from the basic laser frequency of $10^{14} \mathrm{~Hz}$.

Full-field methods, such as particle tracking or image velocimetry (PTV \& PIV), have had only limited success at characterizing 3-D flows. These techniques use particles that are easily trapped within microfluidic devices. These particle techniques also suffer from heavy computational demands and are prone to errors related to correlating particles from one image to another for complex flows with substantial velocity through the plane of illumination. Furthermore, due to inertial effects, particles are often ejected out of high vorticity regions where information cannot be obtained. Additionally, objects or obstacles within or near regions of interest within the flow field can also deplete the area of the seeded particles, making accurate flow measurements difficult.

This non-invasive, high-resolution, molecular tagging velocimetry technique that we are developing obtains direct velocity field measurements of complex aerodynamic and hydrodynamic flows. The technique uses fluorescent or colored molecules as flow tracers, does not have particle seeding requirements, and is well suited to capture the full flow field instantaneously, in three dimensions. Velocity gradients are obtained from the detailed velocity field measurements. From this gradient field, the pressure field of the entire region of interest can be derived from the Navier-Stokes equation using a local modeling technique.

\section{METHOD AND THEORY}

The molecular tagging technique uses a laser to tag fluid elements by changing the electronic states of the tagged molecules. These tagged molecules are then tracked in time. There are two different molecular tagging methods: laser-induced fluorescence/phosphorescence [4] and the photochromic [5] method. When laser-induced fluorescence/phosphorescence molecules are used, the tagging trace is visualized by photon emissions from the trace itself. These emissions are induced by the tagging laser or by the secondary illumination. For the photochromic method, the tagging trace is visualized by the selective absorption of the backlit illumination. These traces are typically observed as a change in color, usually involving a conversion from a colorless to a colored form (e.g., from clear to dark blue). In both cases, a tagging laser is used either to excite the tagging molecule to a long lifetime, higher energy state or to induce a photolysis reaction that activates ( or "uncages") the tagging molecules to a light sensitive dye [6]. Typically, the liquid working concentrations are on the order of ten parts per million (ppm), with the excited molecule having a half-life ranging from microseconds to tens of seconds.

After the fluid is tagged, the displacement of the tagging molecules is typically captured by a CCD. For photochromic and caged molecule methods, another short-duration light source is used to capture the photochromic image or to illuminate the released active dye for fluorescent emission. However, past studies typically used a xenon flash lamp to capture images of the colored tagging molecules. This light source, with its wide band of wavelengths, has limited the optical response range of the photochromic tagging molecules to be mainly in visible. To overcome this limitation, we have replaced the flash lamp with a tunable, pulsed laser source (with a wavelength range of $200-$ $1000 \mathrm{~nm}$ ). The monochromatic and tunable output from the laser allows us to consider molecules that were previously not visible and are only observable in the UV or infrared regions.

In order to analyze a flow field, typically, laser pulses are redirected by beam splitters and lens arrays to tag fluid molecules so that sets of fine lines can be observed within the region of interest. By comparing two consecutive images of these trace 
patterns, at known points in time, the displacement field can be measured and used to estimate the instantaneous velocity field, $\boldsymbol{u}$. Using a local interpolation technique [7], field gradients, vorticity, pressure fields, and all flow variables throughout the field can be determined, with an accuracy of $<5 \%$ of the peak velocities. The vorticity field, $\omega$, is defined as the curl of the velocity,

$$
\omega=\nabla \times u \text {. }
$$

The wall shear stress, $\tau_{w}$, can be determined everywhere within the region of interest as functions of the dynamic viscosity of the fluid, $\mu$, and the gradient of the tangential velocity along the normal direction of the wall, $n$,

$$
\tau_{w}=\left.\mu \frac{\partial u_{l}}{\partial n}\right|_{\text {wall }} .
$$

Furthermore, the first and second velocity gradient fields will be used to estimate the pressure field, $P$. This will be accomplished by solving the Navier-Stokes equations for steady, incompressible, 3-D flow,

$$
\begin{gathered}
\nabla \cdot \rho \boldsymbol{u}=0 \\
\partial \boldsymbol{u} / \partial t+(\boldsymbol{u} \cdot \nabla) \rho \boldsymbol{u}=-\nabla P+\mu \cdot \nabla^{2} \boldsymbol{u}+\rho g,
\end{gathered}
$$

and integrating the resulting pressure gradient field. Here, $\rho$ is the fluid density and $g$ is the gravitational constant. Equation (3a) is the mass continuity condition, and equation (3b) states that the force in the flow direction equals to momentum change of the flow plus the viscous dissipation and the gravitational force.

\section{EXPERIMENTAL DETAILS}

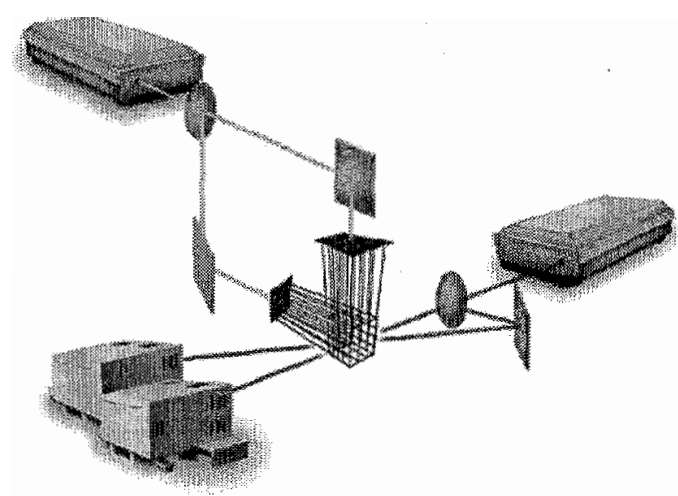

Figure 1. Experimental Set-up for 2-D Molecular Tagging Velocimetry. General Method: 1. Tagging laser generates a short UV pulse which is split and focused into region of interest. 2. Along each line of the resulting grid, UV-sensitive indicators are instantly activated. 3. A second laser, tuned specifically for the activated species, is used to capture the grid pattern at set time intervals. 4. Two views of the 2-D grid are acquired by placing a camera at two positions to stereoscopically capture full 3-D movement. 5. Every aspect of the flow field can be obtained including: instantaneous 3$D$ velocity, vorticity, shear stress and pressure fields.

The experimental setup for achieving $2 \mathrm{D}$ measurement is shown schematically in Figure 1. A Nd:Yag laser is used to provide the UV tagging pulses. The maximum pulse energy is $1000 \mathrm{~mJ} / \mathrm{pulse}$, and the pulse width is $10 \pm 2 \mathrm{~ns}$. The beam diameter is about $1 \mathrm{~cm}$ with operating wavelengths of $266 \mathrm{~nm}$ and $355 \mathrm{~nm}$. The output pulse is split into two beams propagating along two orthogonal directions by a beam splitter, and redirected to the focusing lens arrays by a pair of dielectric coated mirrors. The laser beams focused by these lens arrays will intersect within the region of interest to produce a two-dimensional $10 \times 10$ grid pattern with 100 nodes of intersection. The second laser is a tunable laser light source with a wavelength range of $200-1000 \mathrm{~nm}$. This laser serves as a strobe, and can be tuned to the absorption peak of the excited tagging molecules to capture optimal photochromic change, or to the excitation wavelength of "uncaged" dyes to produce fluorescence emission. A high resolution, $1300 \times 1300$ pixel CCD is used to capture the motion of the grid pattern. A computer and two digital delay generators are used to synchronize the components of the experimental system, including the lasers and the cameras. The computer is also used to record the raw image data from the CCD camera, process the images, and analyze the data to obtain relevant flow field information.

Two photochromic fluid solutions are tested here. The first fluid contains $50 \mathrm{ppm}$ of photochromic dye (1,3,3,-trimethyl-6nitro-indoline-2-spiro-2-2-benzopyrane) dissolved in deodorized kerosene (Shell-Sol 715), the second is an aqueous fluid that contains $100 \mathrm{ppm}$ of a water-based, light sensitive dye.

Two test devices are reported here. A simple, $2 \mathrm{~mm} \mathrm{x} 1 \mathrm{~cm} \mathrm{x}$ $15 \mathrm{~cm}$, rectangular device is used to demonstrate a $2 \mathrm{D}$ grid. A blank microtissue array biosensor is used to demonstrate an actual microfluid application. The biosensor was developed in collaboration with researchers at MIT. It is made of UV transparent acrylic and houses a stainless steel scaffold patterned with an array of $4 \times 10$ microchannels. Each microchannel has a $300 \mu \mathrm{m}$ square cross-section and a $500 \mu \mathrm{m}$ pitch. A schematic of the biosensor is shown in Figure 2. There is one flow inlet and one flow outlet above the scaffold, and an additional flow outlet beneath the scaffold. The inlet flow enters the biosensor and flows into the region above the scaffold. Part of the inlet flow will continue laterally and exit the biosensor from the top outlet, and the remainder of the flow will pass through microchannels and exit the biosensor via the bottom outlet.

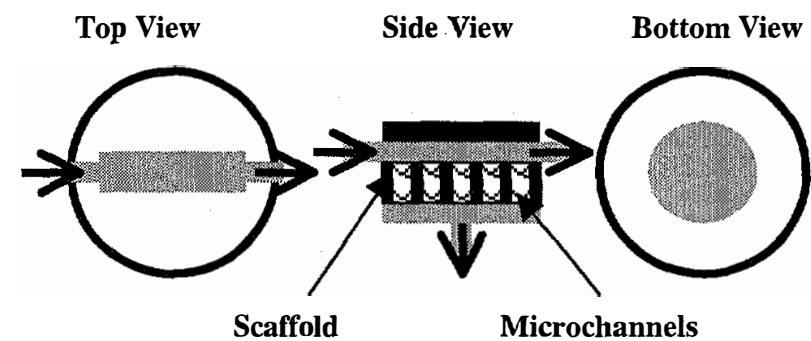

Figure 2 Schematics of a biosensor. The figure shows top, side and bottom views of the device. The arrows indicate inlet and outlets. The top inlet and outlet are horizontal, and the bottom outlet is vertical. The patterned area in the center of the side view is the scaffold, where lighter regions represent microchannels. The top chamber above the scaffold is $2 \mathrm{~mm}$ wide, $6 \mathrm{~mm}$ long, and $3 \mathrm{~mm}$ deep. The diameter of the bottom chamber is $10 \mathrm{~mm}$, and the depth is about $6 \mathrm{~mm}$.

As is shown in the side view, the heart of the biosensor is an array of capillary bed-sized units of "tissue beds" made by selfassembly of dissociated cells from native tissue within the confines of small through-channels created in a silicon or stainless chip by deep-trench etching. Direct measurements of the flow fields through each channel will allow us to design the biosensor for optimal performance. 
The measurement will indicate if there is preferential or unequal tlow distribution along the microchannels, and if there are any "dead zones", where the flow is stagnant and trapped by the local flow geometry. Figure 3 shows how the experiment is conducted to measure the flow rate of each microchannels. A laser line trace is placed right below the scaffold, which holds the tissue array microchannels. The position of the trace at a given delay time, $t_{1}-t_{0}$, is captured by a CCD.

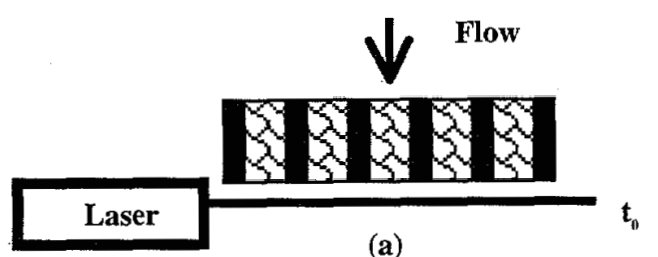

(a)

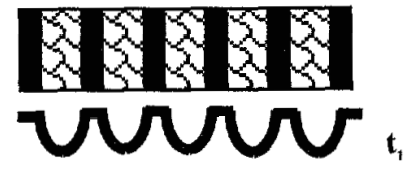

(b)

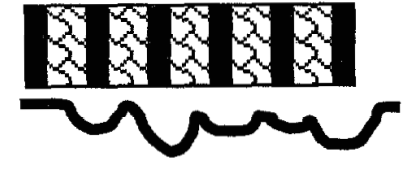

(c)
Figure 3 Schematic demonstration of the flow measurement. (a) A laser line trace is placed directly below the tissue array under a series of microchannels, and is captured at time $t_{0}$ immediately after the tagging pulse. (b) The $2^{\text {nd }}$ trace is captured at a later time $t_{1}$ showing displacement from the initial trace. (c) A case where the flow distribution is unequal.

\section{RESULTS AND DISCUSSIONS}

The kerosene based tagging solution was first tested in a $2 \mathrm{~mm} \times 1 \mathrm{~cm} \times 15 \mathrm{crn}$ rectangular device. A 2-D photochromic grid was generated and is shown in Figure 4. The line-traces were approximately $100 \mu \mathrm{m}$ wide, and inter-trace space spacing is $1 \mathrm{~mm}$. The flow is from the top-right to the bottom-left, the Reynolds number is less than 1.
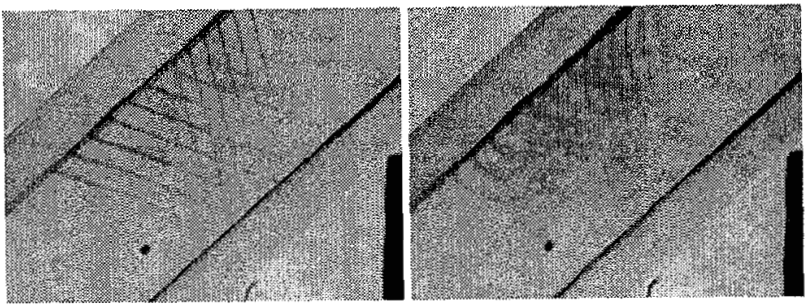

Figure 4. Two-dimensional photochromic grid pattem in a $1 \mathrm{~cm} x$ $2 \mathrm{~mm}$ rectangular cell. (a) immediately after tagging laser pulse and (b) 1 s afterwards. Flow is from top-right to bottom-left. The spacing of the line-traces is $1 \mathrm{~mm}$, and the line-trace width is 0.1 $\mathrm{mm}$. The working fluid is kerosene with $0.005 \% U V$-sensitive dye.

A similar result is obtained using a water-based fluid. This is of particular interest since a lot of biological applications require the use of biocompatible, aqueous fluids. In Figure 5, the optical response of the water-based solution is demonstrated by an array of parallel, $100 \mu \mathrm{m}$ wide traces with a $1 \mathrm{~mm}$ inter-trace spacing.

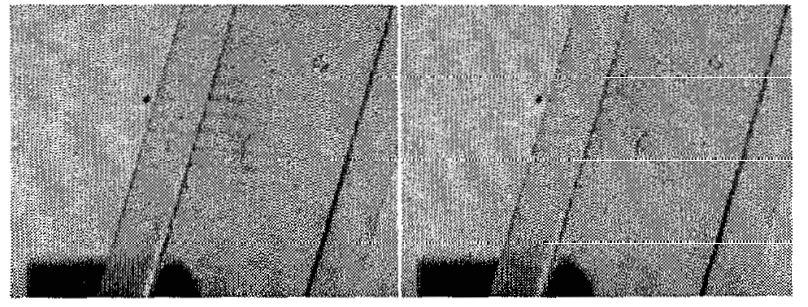

Figure 5. Photochromic traces in a $1 \mathrm{~cm} \times 2 \mathrm{~mm}$ rectangular cell. (a) immediately after tagging laser pulse and (b) 1 s afterwards. Flow is from bottom-left to top-right. The spacing of the linetraces is $1 \mathrm{~mm}$, and the line-trace width is $0.1 \mathrm{~mm}$. The working fluid contains a UV sensitive, water-based dye.

In our most recent work, an array of photochromic line-traces were generated directly beneath the microchannels of the scaffold with a model of the biosensor. The working fluid is kerosene with $0.005 \% \mathrm{UV}$-sensitive dye. A CCD image of the side view of the biosensor is shown in Figure 6(a). The flexible tubing at the top of the picture is the inlet, and the L-shaped channel located at the bottom is the bottom outlet of the biosensor. Another top outlet is on the opposite side of the device, and therefore cannot be seen. The dark thin plate across the image is the stainless steel scaffold with the microchannel array. The scaffold also divides the biosensor into a $2 \mathrm{~mm} \times 6 \mathrm{~mm}$ top chamber, and a $10 \mathrm{~mm} \times 10 \mathrm{~mm}$ bottom chamber. The flow is from top to bottom, and the Reynolds number is approximately 0.3 . The displaced tagging traces 5 seconds after the tagging pulse is shown in Figure 6(b). Again, the initial spacing of the line-traces is $1 \mathrm{~mm}$, and the line-trace width is approximately $0.1 \mathrm{~mm}$.

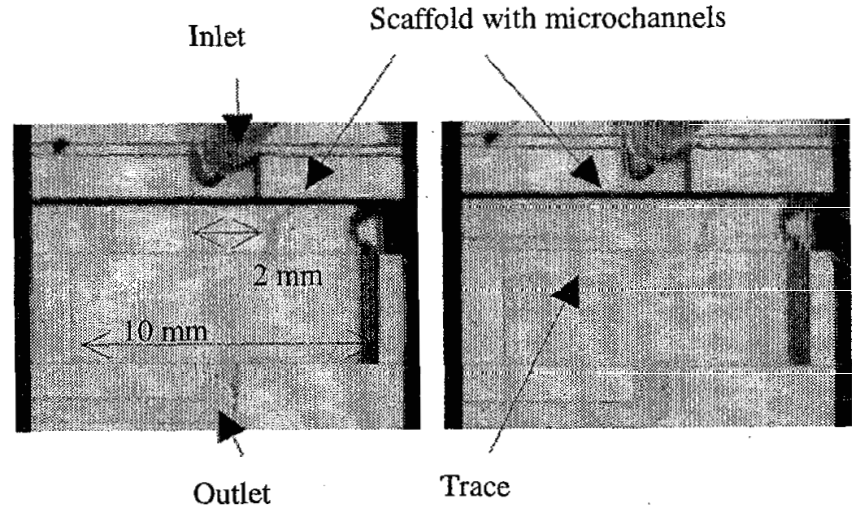

Figure 6. Photochromic traces in a microtissue array biosensor. (a) side view of the testing device. The top inlet feeds the tagging fluid into a $2 \mathrm{~mm}$ wide, $6 \mathrm{~mm}$ long top chamber; while the bottom outlet is connected to a $10 \mathrm{~mm} \times 10 \mathrm{~mm}$ bottom chamber. The two chambers are separated by a stainless steel scaffold with an array of $4 \times 10$ microchannels. A top outlet is on the other side of the device and can not be seen from this image. (b) image of photochromic traces taken 5 seconds after the tagging pulse. The Reynolds number is approximately 0.3. The initial spacing of the line-traces is $1 \mathrm{~mm}$, and the line-trace width is $0.1 \mathrm{~mm}$. The working fluid is kerosene with $0.005 \% \mathrm{UV}$-sensitive dye.

After digital subtraction, the image data from Figure 6 is shown in Figure 7. Note that there are sevcral interesting features. The separate flows out of the four microchannels can be clearly 
seen in this image. Due to an effectively no slip boundary condition at the wall of the top chamber, the flow rate out of the two microchannels on either side of the two center channels is slightly less than the flow rate out of the two center channels. Due to viscous action, the flow profile takes on a more familiar parabola-like shape as the flow travels away from the microchannels. Note that the curved profile becomes wider as the flow gets farther away, showing that the non-zero velocity flow profile expands as it enters the larger bottom chamber. The nonzero flow profile converges as it approaches the narrow outlet at the bottom, while the maximum flow speed at the center profile increases substantially. In addition, note that the trace lines on the side barely move, indicating regions that are "dead zones" where elements in the flow media can be trapped due to extremely long residence times. These dead zones can alter the outcome of the biosensor measurement and compromise it's performance and reliability, as the trapped material may lead to contamination or false readings.

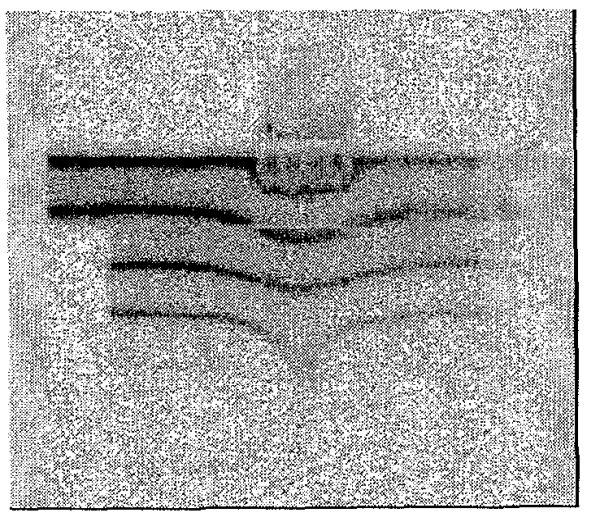

Figure 7. A digitally subtracted image of the photochromic traces in a microtissue array biosensor. There are 4 obvious trace lines shown here. The center of top trace displays the flow profile as it exits the 4 microchannels. The $2^{\text {nd }}$ and $3^{\text {rd }}$ trace shows that the profile is expanded wider and converted to a parabolic shape due to the viscous action. The bottom trace shows an increase of the maximum flow speed at it exits through the narrow outlet.

Only 2D tagging result were reported here. However, with minor additions to the experimental configuration of the measurement system, instantaneous 3-D measurements will be obtained during future work. The 3-D grid will be generated to cover volumes of interest with side dimensions ranging from $5 \mathrm{~mm}$ to several centimeters. The traces can be produced with a thickness below 50 microns for grid patterns. The inter-trace spacing is often $500 \mu \mathrm{m}$ to $1 \mathrm{~mm}$ or less, with an effective resolution that approaches tens of microns using local modeling and image analysis techniques. For single trace applications the trace thickness can approach several microns. The ultimate resolution of the system will also be enhanced by state-of-the art image processing techniques including edge detection and active contour methods. In terms of temporal resolution, the initial and final images are taken from microseconds to seconds apart. By stereoscopically capturing the displacement of this grid with two CCD cameras, or one camera placed at two different locations, the 3-D velocity field can be obtained.

\section{CONCLUSIONS}

Currently, we are in the process of testing various watersoluble and air-based molecular tagging candidates and are developing the flow analysis techniques for vector interpolation and local modeling. Such detailed information is valuable in any effort to develop applications where the fluid flow characteristic have a vital role in effective operation, such as microchip-based biosensor, genetic analysis instruments, micro-pumping and actuation. It may also lead to many product innovations in the fields of drug inhalers, artificial heart valves, and coronary and vascular bypass grafts.

\section{ACKNOWLEDGMENTS} work

The authors acknowledge DARPA DSP for support of this

\section{REFERENCES}

1. P. Gravesen, J. Branebjerg, and O. Sondergard, "Microfluidics - A Review", Journal of Micromechanics, 3, 168 (1993).

2. M. T. A. Saif and N. C. MacDonald, "Measurement of Forces and Spring Constants of Mocroinstruments", Review of Scientific Instruments, 69, 1410 (1998)

3. Y. Cho, A. P. Pisano, and R. T. Howe, "Viscous Damping Model for Laterally Oscillationg Microstructures", Journal of Microelectromechanical Systems, 3, 81 (1994)

4. B. Stier and M. M. Koochesfahani, "Molecular Tagging Velocimetry Measurements in Gas Phase Flows", Experiments in Fluids, 26, 297 (1999)

5. R. E. Falco and C. C. Chu, "Measurement of Twodimensional Fluid Dynamic Quantities Using a Photochromic Grid Tracing Technique", SPIE, 814, Photomechanics and Speckle Metrology, 706, 1987

6. J. E. Guilker, K. R. Gee, P. A. McMurtry, J. C. Klewicki, "Use of Caged Fluorescent Dyes For The Study of Turbulent Passive Scalar Mixing", Experiments in Fluids, 21, 237 (1996)

7. H. Park, J. A. Moore, O. Trass, M. Ojha "Laser photochromic velocimetry estimation of the vorticity and pressure field, twodimensional flow in a curved vessel" Experiments in Fluids 26,55 (1999) 